\title{
Type Ia Supernovae and the Uncertainties in their Progenitor Evolution
}

\author{
J. S. W. Claeys ${ }^{1}$, O. R. Pols ${ }^{1}$, and R. G. Izzard ${ }^{2}$ \\ ${ }^{1}$ Department of Astrophysics/IMAPP, Radboud University Nijmegen, \\ PO Box 9010, NL-6500 GL Nijmegen, the Netherlands \\ email: J.Claeys@astro.ru.nl \\ ${ }^{2}$ Argelander-Institut für Astronomie, University of Bonn, \\ Auf dem Hügel 71, 53121 Bonn, Germany
}

\begin{abstract}
We use binary population synthesis to study the main proposed channels leading to Type Ia supernovae, the single degenerate channel (SD) and double degenerate channel (DD). For this purpose, we discuss the progenitor evolution and the influence of the common envelope efficiency, $\alpha_{\mathrm{ce}}$, on the rate of the different channels. Our study demonstrates the large $\alpha_{\mathrm{ce}}$ dependence of both channels, especially for the SD channel.
\end{abstract}

Keywords. supernovae: general - stars: evolution

\section{Introduction}

Type Ia supernovae (SNe Ia) are used as cosmological distance indicators and are important contributors of iron in the universe. It is generally accepted that they are thermonuclear explosions of carbon-oxygen white dwarfs (CO WDs) which approach the critical Chandrasekhar mass. However, their nature is poorly understood. Current binary evolutionary models cannot reproduce the observed rate of Type Ia SNe. We study the two main proposed channels towards SNe Ia, namely the double degenerate (DD, coalescence of two CO WDs) and the single degenerate channel (SD, a CO WD accreting from a non-degenerate companion). We do this by investigating the effect of the parameter describing the common envelope efficiency. We look at the rate and the progenitor evolution of SNe Ia for each channel.

For this paper we use a binary population synthesis (BPS) code, which will be discussed in Section 2, which makes it possible to study large grids of binary models and the effect of uncertain parameters on whole stellar populations. In Section 3 we discuss the general progenitor evolution of the DD and SD channel and the main effects on the total number of systems evolving according to these channels. Section 4 contains the preliminary conclusions.

\section{Binary Population Synthesis}

The calculations are performed with the BPS code based on Hurley et al. $(2000,2002)$ and updated by Izzard et al. (2006, 2009) \& Claeys et al. (in prep.). The common envelope (CE) phase is one of the most uncertain aspects of binary evolution. In the code, we use the $\alpha$-prescription, which considers the energy budget during the CE-phase (Webbink 1984, Hurley et al. 2002). In this prescription there are two unknown parameters, $\lambda_{\text {ce }}$ and $\alpha_{\text {ce }} . \lambda_{\text {ce }}$ describes the mass distribution in the stellar envelope. $\alpha_{\text {ce }}$ describes the common envelope efficiency, namely the fraction of orbital energy that is transferred to the envelope. If $\alpha_{\mathrm{ce}}$ is less than 1, the envelope gains less energy than the loss of orbital energy. If $\alpha_{\mathrm{ce}}$ is higher than 1, the opposite occurs, which would imply an extra energy 
source. For this research $\lambda_{\text {ce }}$ is variable and depends on the type of star and $\alpha_{\text {ce }}$ is taken to be equal to 1 , unless otherwise specified.

In the simulations performed, the initial primary mass ranges from 1 to $12 \mathrm{M}_{\odot}$, and these are distributed according the IMF described in Kroupa et al. (1993). The mass of the companion varies between 0.1 and $12 \mathrm{M}_{\odot}$, and is distributed with a flat mass ratio distribution. The initial separation is between 3 and $10^{4} \mathrm{R}_{\odot}$, distributed flat in $\log a$ (with $a$, the initial separation). To compare the different channels and the effect of $\alpha_{\text {ce }}$, we count the number of systems of SNe Ia events occurring within each channel and divide this by the total number of binaries formed with parameters in the ranges described above. The percentages given in the next section all refer to this ratio as defined above.

\section{Progenitor Evolution}
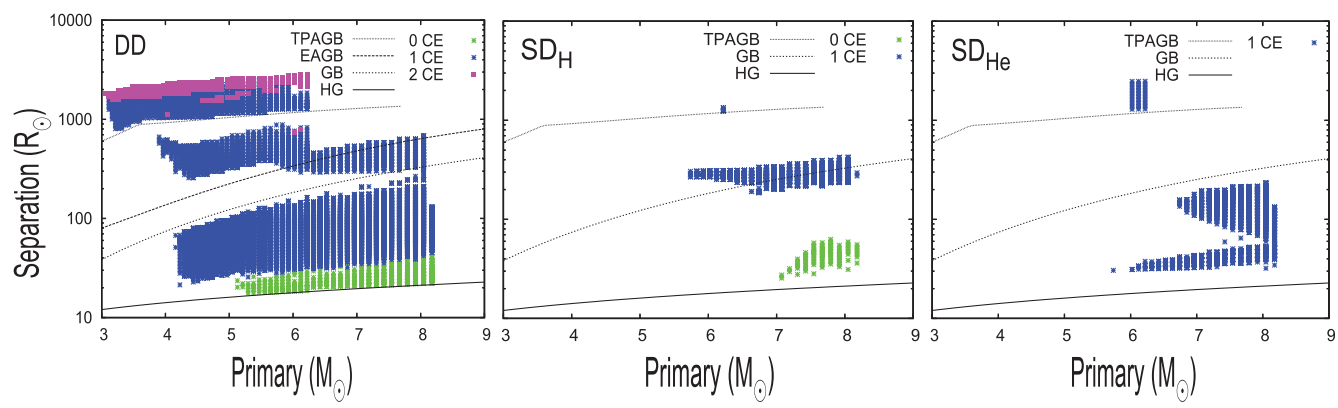

Figure 1. Initial separation versus initial primary mass of the systems evolving to $\mathrm{SNe}$ Ia through the DD-channel (a, left panel) or the SD channel (b, middle panel: with H-rich donors. c, right panel: with He-rich donors). Different colors indicate the number of CE-phases necessary for the system to evolve to $\mathrm{SNe}$ Ia $(2 \mathrm{CE}$ phases $=$ magenta, $1 \mathrm{CE}$ phase $=$ blue, $0 \mathrm{CE}$ phases $=$ green). Different lines indicate the minimum separation for the primary star interaction when in a certain evolutionary stage.

\subsection{Double Degenerate Channel}

It is still under debate whether this channel produces SNe Ia, because early models showed that the merger of two WDs produces an accretion induced collapse instead of an SN Ia (Nomoto \& Kondo 1991). However, population synthesis favors this channel to reproduce the SNe Ia rate. Only recent studies have proven that it is possible to explode the merger product, but as a subluminous SNe Ia (Pakmor et al. 2010). In our code the merger product is assumed to result in an SN Ia. The rate of this channel is determined by the number of systems with $2 \mathrm{CO}$ WDs (with $\mathrm{M}_{\mathrm{WD}, 1}+\mathrm{M}_{\mathrm{WD}, 2} \geqslant \mathrm{M}_{\mathrm{ch}}$ ) in a close enough orbit ( $\lesssim 0.6$ days). Figure 1a shows the initial separations and the initial primary masses of the systems that satisfy these conditions, with the standard assumption discussed in Section 2. In this figure, three main regions can be distinguished, with small, intermediate and large initial separations:

- The smallest initial separations have the first mass transfer phase when the primary is on the Hertzsprung gap (HG), which results in stable mass transfer and a CE phase is avoided. The second phase of mass transfer, from the initially less massive star to its companion (which is now a CO WD), can be stable or unstable depending on the initial separation, resulting in either zero or one CE phase in total.

- The intermediate initial separations have the first mass transfer phase when the primary is on the first giant branch (GB) or early asymptotic giant branch (E-AGB). This is unstable and a CE-phase follows and leaves a He-star behind. This evolves further and 
subsequently a CO WD is formed. The second phase of mass transfer is stable. Hereafter, $2 \mathrm{CO}$ WDs are formed in a close orbit. In order to avoid a merger of the binary during the CE phase, the initial orbit has to be relatively wide, which explains the gap in Figure 1a around $a \sim 100 \mathrm{R}_{\odot}$.

- For the initially widest systems, the first mass transfer phase starts when the primary is on the thermally pulsing asymptotic giant branch (TP-AGB), which is unstable. A CE phase follows, after which a CO WD is immediately formed. Whether the second phase of mass transfer is stable or unstable ultimately depends upon the initial separation, but both situations occur. Two WDs are formed after one or two CE phases. Another gap appears in Figure 1a around $1000 \mathrm{R}_{\odot}$; these systems merge during the CE phase while the primary is on the E-AGB.

Binary systems with primary masses higher than $6.2 \mathrm{M}_{\odot}$ and with the first mass transfer phase during the AGB cannot form a double CO WD system (see Figure 1a), because these systems start RLOF when the core of the primary already consists of oxygen and neon. Therefore, an ONe WD is formed. The merger of an ONe WD with another ONe $\mathrm{WD}$ or a $\mathrm{CO}$ WD is assumed not to form a SNe Ia, but an electron-capture supernova. About $0.3 \%$ of systems in our grid (see Section 2) evolve into SNe Ia according to this channel. This numbers is mainly affected by the common envelope efficiency, $\alpha_{\text {ce }}$. Smaller values of $\alpha_{\text {ce }}$ yield more orbital shrinkage during the CE phase. In the case of $\alpha_{\text {ce }}=0.2$, only $\sim 0.1 \%$ evolves to a SNe Ia according to the DD channel, while for $\alpha_{\text {ce }}=3, \sim 0.4 \%$ evolves into SNe Ia.

\subsection{Single Degenerate Channel}

In the SD channel a WD accretes from a non-degenerate companion and burns the accreted material into carbon and oxygen. The WD burns this material steadily in a limited range of accretion rates, depending on the composition of the accreted material $\left[\sim 10^{-7} \mathrm{M}_{\odot} / \mathrm{yr}\right.$ for a H-rich donor, $\sim 10^{-6} \mathrm{M}_{\odot} / \mathrm{yr}$ for a He-rich donors (Nomoto 1982)]. If the WD accretes at a lower rate, the accreted material is not immediately burnt, but a layer of accreted material is built up which results in a nova outburst. If the material is accreted at too high a rate, the WD cannot burn the material and either a giant envelope is formed around the WD (Nomoto 1982) or the material is blown away due to an optically thick wind from the WD (Hachisu et al. 1996).

Consequently, the rate of this channel depends on the treatment of accretion onto a WD, but also on the number of systems that are in a close enough orbit to provide stable mass transfer towards the WD. For this work we use a prescription based on Hachisu et al. (1996). In the following paragraphs we discuss both types of donors in more detail.

\section{H-rich donors}

These donors can be in any stage of evolution, varying from the main sequence (MS) to the AGB, with the separation at the moment of formation of the WD depending on the type of donor star (see e.g. Meng \& Yang 2010). Three main regions can be distinguished depending on the initial separation (see Figure 1b):

- The initially closest systems have the first phase of RLOF when the primary is on the HG, which results in stable mass transfer, and after this phase a CO WD is formed in a close orbit. These initially close systems produce mainly CO WD with MS donors, but also some systems end in a wider orbit and an SN Ia is formed with a GB donor.

- Intermediate orbit systems start the first mass transfer phase during the GB or at the end of the HG. This results in unstable mass transfer and a CE is formed. These systems produce SNe Ia mainly by MS donors. 
- The few widest systems, with an initial separation around $1000 \mathrm{R}_{\odot}$ and primary mass $\sim 6 \mathrm{M}_{\odot}$, start mass transfer during the TP-AGB phase, which is unstable and a CE-phase follows. These systems will produce Type Ia SNe with GB-donors.

Only $\sim 0.02 \%$ of systems in the grid (see Sect.2) evolves into SNe Ia according to this channel. This channel has a large dependence on $\alpha_{\mathrm{ce}}$. In the case of a large value fewer systems are brought in a close enough orbit and for $\alpha_{\text {ce }}=3$ only $\sim 0.001 \%$ evolve to $\mathrm{SNe}$ Ia according to this channel. For smaller values of $\alpha_{\mathrm{ce}}$ more systems are progenitors of the SD channel, e.g. $\alpha_{\text {ce }}=0.2$ result in $\sim 0.1 \%$ systems that evolve through the SD channel with H-rich donors.

\section{$\underline{\text { He-rich donors }}$}

These donors are evolved stars, originating from relatively massive stars $\left(\gtrsim 4 \mathrm{M}_{\odot}\right)$, with a relatively massive He-core $\left(\gtrsim 1 \mathrm{M}_{\odot}\right)$. Two main formation scenarios can be distinguished:

- In the case of a large initial separation and initial mass ratio close to one, the first mass transfer phase starts when the two stars are evolved and the primary is a TP-AGB. The mass transfer phase is unstable and a CE follows, leaving behind a CO WD and a He-star in a close orbit, so that the He-star can have stable RLOF to the WD (occurs only in $4 \%$ of the systems of the SD channel with a He-rich donor).

- If the two stars have a small initial separation and are less equal in mass, the first mass transfer phase is stable. After this phase, a more massive MS-star is left behind in an orbit with a CO WD. The MS star evolves, but because of the large mass difference mass transfer is unstable. After the CE, a He-star with a CO WD in a close orbit remains (occurs in $96 \%$ of the systems of the SD channel with a He-rich donor).

Only $\sim 0.02 \%$ of the systems in our grid (see Section 2) evolve into SNe Ia according to this channel in the case of $\alpha_{\text {ce }}=1$. In the case of a lower value of $\alpha_{\text {ce }}$, e.g. 0.2 , the number of systems increases to $\sim 0.04 \%$. In the case of $\alpha_{\text {ce }}=3, \sim 0.02 \%$ also evolve to SNe Ia according to this channel.

\section{Conclusions}

The progenitor evolution of SNe Ia for the different channels depends strongly on the CE evolution. Variation of $\alpha_{\text {ce }}$ has a different effect on the SD and DD channels. The lower the value of $\alpha_{\text {ce }}$, the smaller the number of systems becoming SNe Ia according to the DD channel, and the larger the number of systems evolving according to the SD channel. The SD channel with H-rich donor is most affected by this uncertain factor; only for low values of $\alpha_{\text {ce }}$ is the rate comparable with the other channels. The large influence on both channels indicates that it is worthwhile to get better restrictions on $\alpha_{\text {ce }}$, by both observations and simulations of the CE phase.

\section{References}

Hachisu, I., Kato, M., \& Nomoto, K. 1996, ApJ, 470, L97

Hurley, J. R., Pols, O. R., \& Tout, C. A. 2000, MNRAS, 315, 543

Hurley, J. R., Tout, C. A., \& Pols, O. R. 2002, MNRAS, 329, 897

Izzard, R. G., Dray, L. M., Karakas, A. I., Lugaro, M., \& Tout, C. A. 2006, A\&A, 460, 565

Izzard, R. G., Glebbeek, E., Stancliffe, R. J., \& Pols, O. R. 2009, A\& A, , 508, 1359

Kroupa, P., Tout, C. A., \& Gilmore, G. 1993, MNRAS, 262, 545

Meng, X. \& Yang, W. 2010, ApJ, 710, 1310

Nomoto, K. 1982, ApJ, 253, 798

Nomoto, K. \& Kondo, Y. 1991, ApJ, 367, L19

Pakmor, R., Kromer, M., Röpke, F. K., et al. 2010, Nature, 463, 61

Webbink, R. F. 1984, ApJ, 277, 355 